\title{
Robust Helical Path Separation for Thickness Mapping of Pipes by Guided Wave
} Tomography

\author{
P. Huthwaite, M. Seher
}

\begin{abstract}
The pipe wall loss caused by corrosion can be quantified across an area by transmitting guided Lamb waves through the region and measuring the resulting signals. Typically the dispersive relationship for these waves, which means that wave velocity is a known function of thickness, is exploited which enables the wall thickness to be determined from a velocity reconstruction. The accuracy and quality of this reconstruction is commonly limited by the angle of view available from the transducer arrays. These arrays are often attached as a pair of ring arrays either side of the inspected region, and due to the cylindrical nature of the pipe, waves are able to travel in an inifinite number of helical paths between any two transducers. The first arrivals can be separated relatively easily by time gating, but by using just these components the angle of view is severely restricted. To improve the viewing angle, it is necessary to separate the wavepackets. This paper provides an outline of a separation approach: initially the waves are backpropagated to their source to align the different signals, then a filtering technique is applied to select the desired components. The technique is applied to experimental data and demonstrated to robustly separate the signals.
\end{abstract}

\section{Index Terms}

tomography, guided wave, Lamb wave, signal separation, helical paths

Manuscript received XXXXXXX X, XXXX.

All authors are with the Department of Mechancal Engineering, Imperial College, London SW7 2AZ, United Kingdom (email: p.huthwaite@imperial.ac.uk) 


\section{INTRODUCTION}

The management of corrosion presents a significant challenge to the petrochemical industry. Corrosion will cause pipe wall loss, and accurately quantifying the minimum remaining wall thickness is vital to estimating life and establishing whether the pipe can continue to be used safely. Thickness can traditionally be measured through a point-by-point ultrasonic thickness gauge approach, but this is laborious and requires access to all points on the surface. This latter requirement is a particular challenge since corrosion is more likely to occur where water can collect near the surface, which typically happens at inaccessible locations such as supports. Low frequency guided waves [1] can be used to inspect long sections of pipes (typically tens of metres) without requiring access to all points on the surface. The principle is that the guided waves reflect from any defects present and the reflections can be used for detection and location. These systems, however, do not provide good depth sizing capabilities and further, more local inspections are needed to determine size.

A solution has been proposed through guided wave tomography, where guided waves (typically Lambtype modes) travelling within the pipe wall interact with the defect and are measured by an array [2]. A common approach is to produce a map of velocity for all points the waves interact with, then use the dispersive properties of the guided waves to convert the velocity values into an equivalent thickness map; see for example [3]-[8]. The production of such maps is often achieved using a ray approximation, which just requires the arrival times of the different signals as an input [2], [7], [9]-[14]. It has been shown that higher resolution can be achieved through approaches which account for diffraction [8], [15], [16], but these need access to the full wavepacket rather than just the arrival times. Other techniques include the existance of guided wave mode cutoffs, which are frequency-thickness combinations below which certain waves cannot propagate; by analysing the measured waves which have been transmitted in each case it is possible to identify how thick the plate is [17], [18].

However, despite pipe corrosion being a significant application area for this technique, the majority of research has focused on the inspection of flat plates, with only limited work being performed on pipes; exceptions include [7], [14], [19], [20]. The concept considered in this paper is for a pair of parallel ring arrays of transducers to be placed approximately 1-2 circumferences apart either side of the inspected region, as shown in Fig. 1(a); this is considered the most practical for inspecting under a wide variety of supports, although it is acknowledged that alternative configurations are possible (e.g. [19], [21] have considered a line of transducers placed along the meridian). The goal is to use the measured data from the transducer arrays to reconstruct the thickness of the region between the two rings as accurately as 


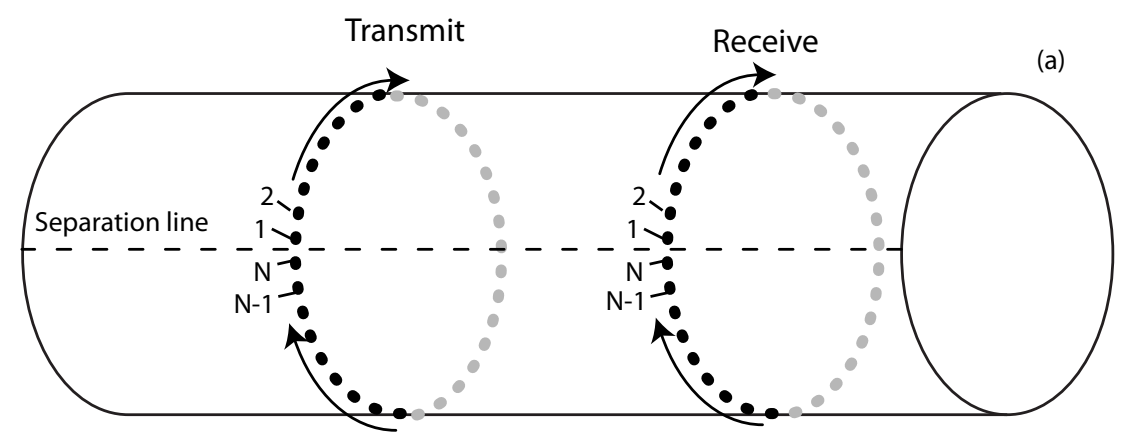

(b)

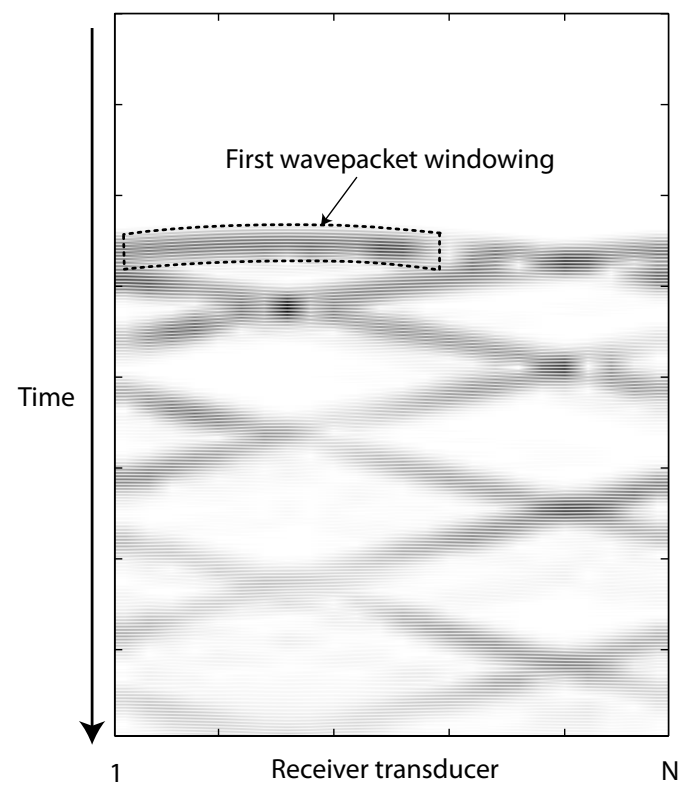

Figure 1. (a) A schematic diagram of the pipe guided wave tomography configuration considered in this paper. (b) presents a 'B-scan' of all the signals, from a numerical model of the pipe, measured by the receiver ring for a particular source - in this illustration source 8 of $N=24$. This illustrates the different helical paths present in the measured signals. One approach for processing is to window to remove all but the initial wavepackets, as marked in the diagram, but there is a significant amount of information discarded through this approach which could be exploited to improve the images.

possible.

An important aspect is that due to the cyclic nature of the pipe geometry, an infinite set of 'helical' paths exists between each source and receiver pair, and the waves from each of these paths will be superposed in the measured time traces. This is illustrated in Fig. 1(b). These signals need to be separated prior to imaging. One simple solution, shown, is to window the signals to remove all but the first wavepacket, however, as acknowledged in [19], this will significantly reduce the range of angles available and hence 
reduce the system's ability to generate accurate reconstructions. Instead, it is of interest to separate out signals even when they overlap, and exploit the additional information in the reconstructed images. Reference [7] appears to extract arrival times from multiple helical signals, but there is no explanation provided for how this is achieved, and to the knowledge of the authors there are no papers which have published any solutions to separate wavepackets for this problem.

Similar problems have been investigated, however. Within guided wave tomography, if multiple Lamb waves are excited and measured, rather than just using a single mode, they can overlap and approaches are needed to separate these out. Reference [4] suggested the use of wavelets to achieve this when time-based separation is not possible, however this only allows for the extraction of arrival times rather than the full wave signals; a similar wavelet technique was also investigated in [22]. A similar approach exploring the use of the discrete wavelet fingerprint (DWFP) technique for this problem is covered in [12], [23]. Other approaches in the separation of different Lamb wave modes include the use of chirplet matching pursuits and mode correlation [24]. In [25] chirp excitations are used to generate broadband signals within a plate-like specimen, which can then subsequently be post-processed with filtering to select the desired mode by changing the frequency range.

This paper presents a solution to helical path separation for guided wave tomography which should be robust to systematic/correlated errors and uncorrelated noise typically present in the measurements, and should also perform well across a range of different corrosion defects. Importantly, this should also allow entire wavepackets to be separated rather than just producing arrival times, since this is important to maximise resolution in the reconstructions. The solution should also produce good results with the subsampled ( $>\lambda / 2$ transducer spacing, where $\lambda$ is the wavelength) arrays typically used. The proposed approach is demonstrated with experimental data, confirming its performance. Section II outlines the full theory used in the helical path separation approach. This approach is subsequently validated with experimental data in Sec. III and conclusions are drawn in Sec. IV.

\section{THEORY}

\section{A. Background}

Lamb waves are a class of ultrasonic guided waves which propagate in elastic plates. They can be considered as an infinite set of bulk waves repeatedly reflected from the top and bottom surfaces, superposed into a single waveform. At most frequencies the Lamb waves are dispersive, as shown in Fig. 2, which is a property frequently exploited by guided wave tomography. The phase and group velocities are functions of the frequency-thickness product, so if frequency is fixed, a change in thickness 

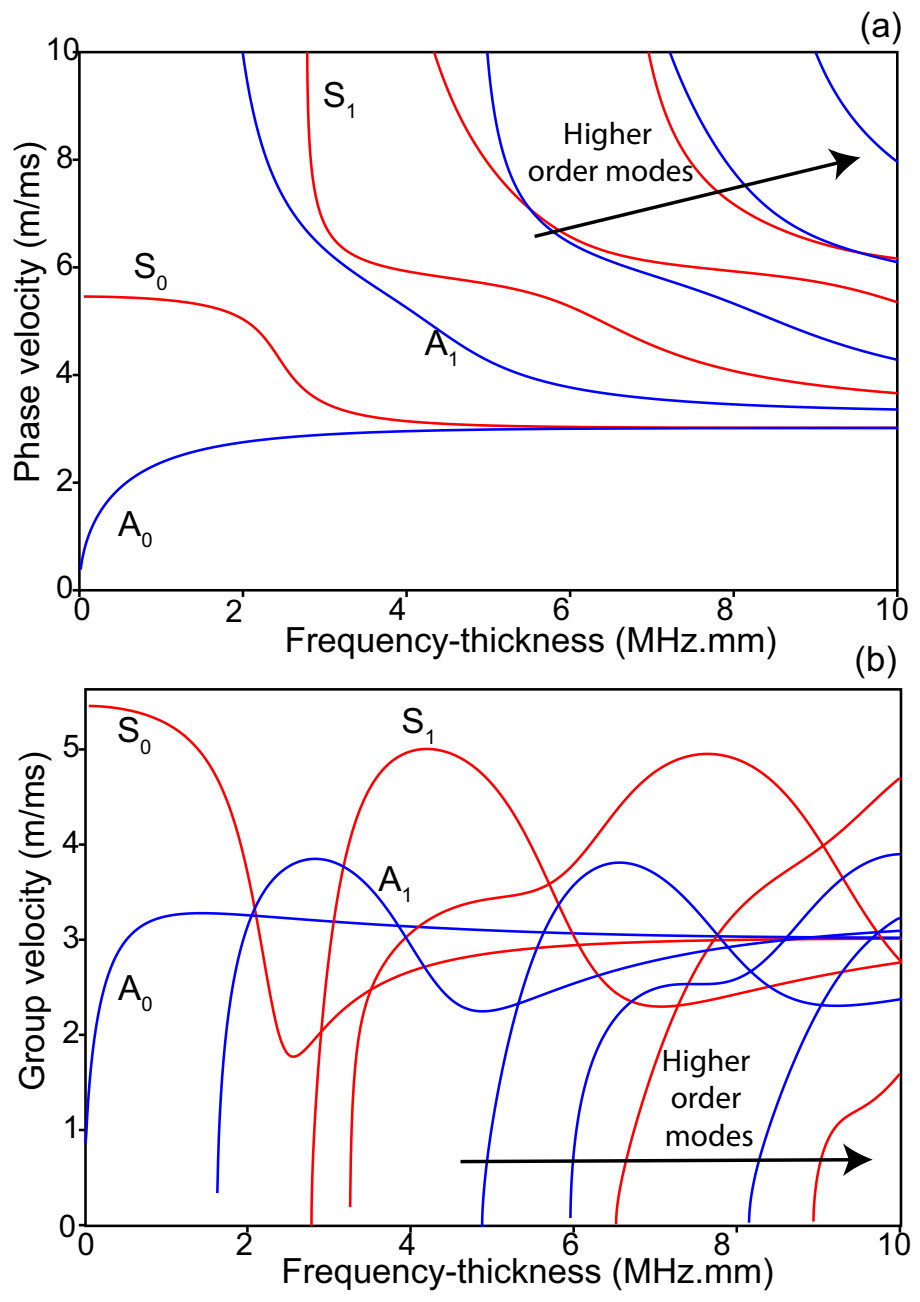

Figure 2. Media-Color 1 Dispersion curves for Lamb waves in plates. (a) presents phase velocity as a function of frequency, and (b) shows group velocity.

will result in a known change in velocity. Therefore, if a reconstruction of velocity is produced, this can subsequently be converted back to thickness; an evaluation of the accuracy of the assumptions made in this approach is given in [26]. While the Lamb waves are defined for flat plates, rather than curved pipe walls, in this paper the wall curvature will be considered negligible; this can be shown to be a valid assumption if the thickness is less than around $10 \%$ of the radius [27], [28], which is the case for the majority of pipes of interest.

The theory of the separation approach is derived for a 'defectless' pipe, i.e. any distortion to the signals caused by the defect will be ignored. However, the separation algorithm has tolerance for such 
distortions to the wavefield, and it should also be relatively unaffected by noise or systematic errors (such as a misalignment of the transducer ring). The algorithm is later tested with full experimental guided wave data to confirm this. Since the pipe has no defects, a 2D acoustic model provides an accurate representation of the guided waves, with the phase velocity of the acoustic medium at each frequency being set to that of the Lamb wave.

As illustrated in Fig. 1(a), the chosen configuration has $2 N$ transducers in total, with $N$ transducers in each of the transmit and receive rings. The transducers within each ring are numbered 1 to $N$, with the numbering aligned between the two rings. The jump in the numbering from $N$ to 1 marks the separation line from which unwrapping of the pipe begins. The separation line is marked in Fig. 1(a), and from this the pipe is separated and flattened into a plate as illustrated in Fig. 3(a). A coordinate system is defined for this flattened arrangement with $x$ in the former circumferential direction and $z$ in the former axial direction. Since waves can no longer travel across the separation line, the effect of the pipe's cyclical nature must be reproduced by replicating the flattened section multiple times and attaching these to the edges; this replication can be extended out in both directions to give an infinite domain, as shown in Fig. 3(a). Considering a source $a$ and receiver $b$ on the pipe, the equivalent signal can be generated on the flattened plate by exciting a single source at transducer $a$ within the unreplicated (order 0 ) section, then superposing the measured signals corresponding to all the receivers marked in Fig. 3(b), i.e. all the receivers which correspond to replications of $b$. It is observed that the source transducer need not be replicated since the full set of helical paths is already captured by the replicated receivers.

The separation of signals from $M$ replications, referred to as orders, is considered. The jump in transducer numbering from $N$ to 1 acts as a delimiter between the orders. The 'zeroth order' signals are measured by the receiver array on the flattened plate directly opposite the sources; from this, the negative orders are measured by replications of the receiver array which extend to the left side as shown in Fig. 3 and the positive orders are measured by replications to the right. Throughout this paper, $M$ will be assumed to be odd, so orders from $-(M-1) / 2$ to $(M-1) / 2$ should be extractable.

The target of this paper is develop a process to undo the superposition process which occurs between the replicated sets of receiver transducers. The unseparated, measured time traces can be expressed as

$$
\phi_{r, s}(t)=\sum_{m=0}^{M-1} \psi_{r+N m, s}(t)
$$

where the ideal, perfectly separated time traces have been expressed in the form $\psi_{\tilde{r}, s}(t)$ where $s$ is the source (from 1 to $N$ ), $\widetilde{r}$ is the 'separated' receiver (numbered from 1 to $N M$ ). While this is expressed in 

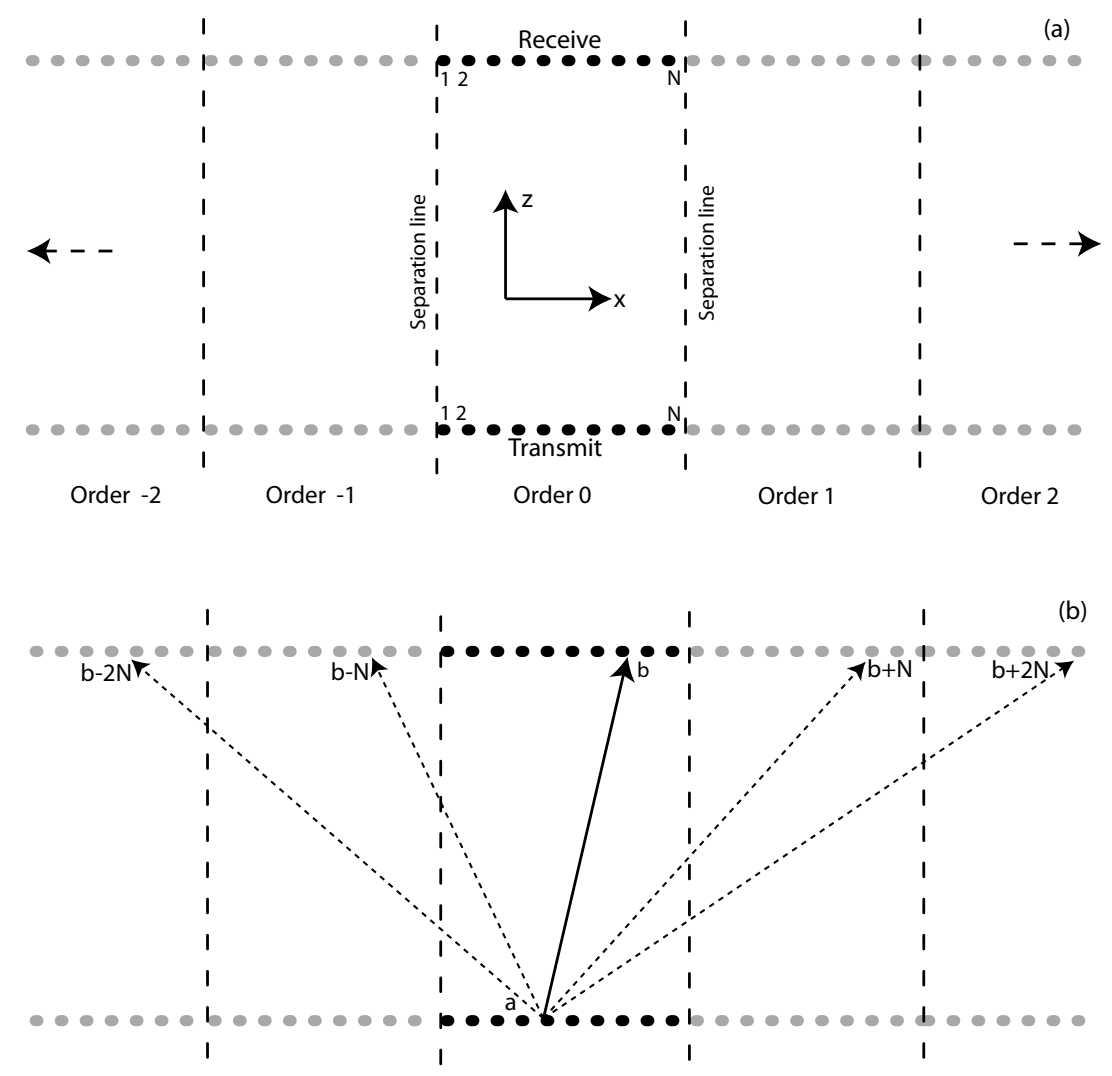

Figure 3. (a) Representation of the pipe wall in the flattened configuration. Order 0 is the section unwrapped from the pipe shown in Fig. 1(a) and other orders are added to both sides to replicate the cyclic nature of the pipe. (b) shows the helical paths that can exist between a source $a$ and a receiver $b$; the measured signal at $b$ on the pipe will be a superposition of the signals from each path marked.

the time domain, it is equally valid in the frequency domain for Fourier-transformed data due to linearity.

\section{B. Helical path separation}

Two features are exploited to identify and separate the different orders in the measured signals. Firstly, as illustrated in Fig. 1(b), the wavepackets generally arrive at different times, so a degree of separation can be achieved by time-windowing the signals. Secondly, the different helical signals have different incident angles when they intersect the array, which provides another method for separating out the orders. An infinite plane wave in the flattened, replicated domain is considered

$$
u=A \exp \left[i\left(k_{x} x+k_{z} z\right)\right]
$$


where $A$ is an arbitrary complex constant and $k_{x}$ and $k_{z}$ are wavenumber magnitudes in the $x$ and $z$ directions respectively. If this was sampled by the separated array in the $x$ direction, the measurements would be

$$
\psi_{\tilde{r}, s}=B \exp \left(i k_{x} \tilde{r} \delta m\right)
$$

where $B$ is a second complex constant, accounting for the absolute $x$ and $z$ position of the array and incorporating $A$, and $\delta m$ is the spacing between the transducers in the x direction in the flattened domain. To separate this signal from any other plane waves which may be present, a discrete Fourier transform can be performed across the receivers (from now on referred to as a DRFT, discrete receiver Fourier transform) and then filtered to remove all components apart from $k_{x} \delta m$, exploiting the fact that $k_{x}$ is a function of incident angle. Note that for clarity throughout this paper, 'components' will exclusively be used to refer to the components of a Fourier transform, rather than the signals received from different helical paths, which will be referred to as orders.

There are issues associated with exploiting this approach for separating helical paths, however. The wavefronts will not be planar; at best they will be hyperbolic, but they will also be affected by any distortions caused by defects or experimental errors in the data. Secondly, if the array is subsampled (i.e. $k_{0} \delta m>2 \pi$ where $k_{0}=\sqrt{k_{x}^{2}+k_{z}^{2}}$ ), aliasing will occur, so multiple plane waves can map to the same frequency component. This makes differentiating the incident wavepaths by this approach alone challenging. One solution to address the non-planar wavefront issue is to apply a window to the signals to select a particular time and set of receivers, to minimise the curvature of the wavefront, then apply the DRFT to this. Typically, this region of interest will be where two waves intersect, since these cannot be separated by their different arrival times. The issue is that the DRFT of this small section will be relatively broadband since the window size is limited (caused by the Gabor 'resolution' limit of short-time Fourier transforms), as opposed to the sharp spike which would be seen for a perfect infinite plane wave. This means that separating out two signals which could contain similar DRFT components due to aliasing is likely to be challenging.

To bypass this issue, a new technique is developed to maximise the length to which the DRFT is applied as much as possible, to improve the resolution of the DRFT output, while avoiding the effects of wavefront curvature. To achieve this, firstly the measured transducer array is replicated $M$ times to account for the different orders

$$
\phi_{r+N m, s}^{(2,1)}=\phi_{r, s}^{(1,1)}
$$



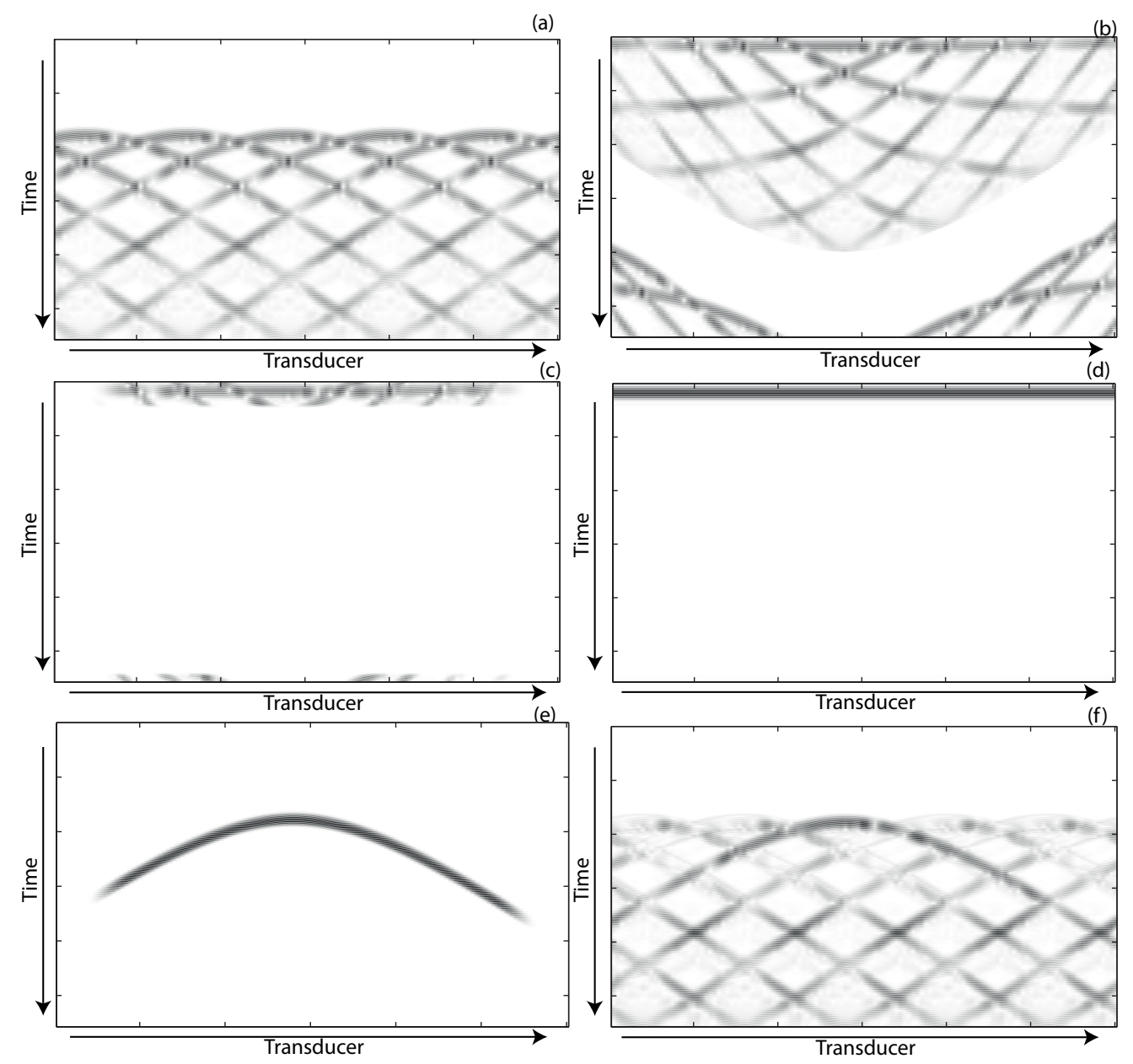

Figure 4. Stages for one iteration of the helical separation algorithm. Here, $N=24$ and $M=5$. This is simulated data from a finite element model of the full pipe experimental setup described in Sec. III; note that dispersion correction by the method outlined in [29] has been applied to these signals. (a) shows the signals received across all transducers, having been replicated five times. In (b), the wavefronts have been backpropagated to the source assuming a homogeneous propagation medium so that the wavefronts are aligned. In (c) a window has been applied to remove the majority of the unwanted modes. In (d) filtering via the DRFT process has been applied, removing all but the first Fourier component (since this is the first iteration, only the uniform, DC, component is kept), and this is propagated forward, as illustrated in (e). In stage (f), the cleaned signal of (e) has been shifted across the different modes (i.e. translated horizontally) and subtracted from all the helical modes in (a) with the exception of the central mode, which is the mode to be extracted. This can then be used in place of (a) for the next iteration except more DRFT components can be kept since the data is cleaner. 
where the superscript $(p, j)$ will be used to indicate the wavefield variable $\phi$ at the $p$ th stage of the algorithm, for the $j$ th iteration, and $m$ will be varied across the orders $-(M-1) / 2$ to $(M-1) / 2$. This replication is shown in Fig. 4(a). Subsequently, the waves are backpropagated (using $\widehat{\phi}$ rather than $\phi$ to signify that this is performed in the frequency domain) such that the central wavefront becomes aligned

$$
\widehat{\phi}_{\tilde{r}, s}^{(3,1)}=\widehat{\phi}_{\tilde{r}, s}^{(2,1)} \exp \left[-i k(\omega) x_{\tilde{r}, s}\right]
$$

where $k(\omega)$ is the wavenumber for each frequency $\omega$ (which can be calculated easily from the dispersion curves) and $x_{\tilde{r}, s}$ is the distance from the receiver back to the separated source; this is illustrated in Fig. 4(b). This backpropagation approach assumes that the waves have propagated through a plate with uniform thickness equal to that of the nominal pipe wall thickness. Since the wavefront of interest is now nearly uniform across the different receivers, it is selected by firstly windowing the signals to remove all orders that arrive at other times

$$
\phi_{\tilde{r}, s}^{(4,1)}=\phi_{\tilde{r}, s}^{(3,1)} w^{\text {time }}(t) w_{\tilde{r}}^{r e c}
$$

where $w^{\text {time }}(t)$ is a window to capture the whole of the signal of interest but remove the majority of the other unwanted modes, and $w_{\tilde{r}}^{r e c}$ is a window in the receiver transducer direction. For $w^{\text {time }}(t)$, a suitable window is defined as

$$
w^{\text {time }}(t)= \begin{cases}0 & t>t_{2} \cap t \leq t_{3} \\ 1 & t \leq t_{1} \cup t>t_{4} \\ \frac{1}{2}\left[1+\cos \left(\frac{\pi\left(t-t_{1}\right)}{t_{2}-t_{1}}\right)\right] & t>t_{1} \cap t \leq t_{2} \\ \frac{1}{2}\left[1-\cos \left(\frac{\pi\left(t-t_{3}\right)}{t_{4}-t_{3}}\right)\right] & t>t_{3} \cap t \leq t_{4}\end{cases}
$$

where $t_{1}<t_{2}<t_{3}<t_{4}$ define the start and end points of a cosine smoothed window. A smoothing length $t_{2}-t_{1}=t_{4}-t_{3}=3 / f_{0}$ was chosen, where $f_{0}$ is the centre frequency. $t_{1}=7 / f_{0}$ to extend beyond the length of the 5 -cycle toneburst input, and $t_{4}=t_{\max }-2 / f_{0}$, where $t_{\max }$ is the total length of the time traces. The signals from this are illustrated in Fig. 4(c); the purpose for including the end of the signals, as seen at the bottom of the figure, is because the backpropagation has resulted in some of the signals 'wrapping around' from the start to the end. The values of $t_{1}, t_{2}, t_{3}$ and $t_{4}$ can be defined easily for the majority of configurations using the approach above; if a different signal length is used the window can be extended or shortened accordingly. It should be noted that the values are independent of the defect itself which will in general be unknown at this point. A similar window is applied in the 
receiver direction

$$
w_{\tilde{r}}^{r e c}= \begin{cases}0 & |\tilde{r}|>N(M+1) / 2-H \\ 1 & |\tilde{r}|<N(M+1) / 2-2 H . \\ \frac{1}{2}\left[1+\cos \left(\frac{\pi(|\tilde{r}|-N(M+1) / 2+H)}{H}\right)\right] & \text { otherwise }\end{cases}
$$

This smoothing window is necessary to minimise spectral leakage which would otherwise occur with the DRFT. Subsequently, the DRFT is performed

$$
{\overline{\phi^{(4,1)}}}_{q, s}=\mathfrak{D}\left(\phi_{\tilde{r}, s}^{(4,1)}\right)
$$

where $\mathfrak{D}$ represents the DRFT operator, $q$ indicates the order of each Fourier component and the bar $\bar{\phi}$ has been used to indicate the transformed state. Then

$$
{\overline{\phi^{(5,1)}}}_{q, s}= \begin{cases}{\overline{\phi^{(4,1)}}}_{q, s} & |q| \leq G \\ 0 & |q|>G\end{cases}
$$

where $G$ defines the limit of the components selected. At the first iteration, $G=0$ is used to select just the first component, and the result is shown in Fig. 4(d). Clearly here, the effect of the defect in the signal has been removed because very few components have been included. On the other hand, if too many components were included, not all parts from the other helical paths will be removed.

A conservative iterative approach is used instead to select the particular helical order. In this, orders which are very likely to belong to other helical paths are removed, which will clean up the order of interest, while still leaving some noise from the orders which were not removed. Then, since the helical order of interest is cleaner, there is more confidence over which signals are in which helical path, so more components can be subtracted off. In practice, the approach is to take the lowest frequency component of the aligned signal, illustrated in Fig. 4(d), i.e. the components which are uniform across the receivers. This can be forward propagated

$$
\widehat{\phi}_{\tilde{r}, s}^{(6,1)}=\widehat{\phi}_{\tilde{r}, s}^{(5,1)} \exp \left[i k(\omega) x_{\tilde{r}, s}\right]
$$

as shown in Fig. 4(e). This can now be used to clean up the signal for the central mode, by minimising the other modes as much as possible. To do this, the waveform in Fig. $4(\mathrm{e}), \phi_{\tilde{r}, s}^{(6,1)}$, is shifted horizontally, by translating in the transducer direction by an integer multiple of $N$, to line up with each of the $M-1$ other modes in Fig. 4(a), and subtracted from each one in turn. Mathematically this is represented as

$$
\phi_{\tilde{r}, s}^{(7,1)}=\phi_{\tilde{r}, s}^{(1,1)}-\phi_{(\tilde{r}+m N) \bmod (N M), s}^{(6,1)}
$$


with $m$ varied from $-(M-1) / 2$ to $(M-1) / 2$ but skipping zero. The result is illustrated in Fig. 4(f). This process has reduced the amplitude of the unwanted modes, while avoiding the removal of any components which could be associated with the defect. The process can then be iterated multiple times, taking

$$
\phi_{\tilde{r}, s}^{(2, j+1)}=\phi_{\tilde{r}, s}^{(7, j)}
$$

with $G$ being increased each time to take more components from the DRFT since the signal is cleaner and less likely to contain signals from other modes. The exact number of components extracted can be tuned to a particular array configuration, but in the configuration considered here $(M=5, N=24$, with an array separation distance of $25 \lambda$ and pipe circumference of $25 \lambda) G=0,1,2,3,4$ was used to extract and subtract components from the other modes each time.

Having removed the majority of the unwanted signals through this process, there will often still be a small amount of remaining noise from other orders in $\phi_{\tilde{r}, s}^{(7, J)}$, the signals after the final, $J$ th iteration. Therefore, a final 'cleaning' step is performed. In this, the stages up to $\phi^{(3, J+1)}$ are performed as before. A window is applied to remove any remaining signals away from the order of interest

$$
\phi_{\tilde{r}, s}^{(4, J+1)}=\phi_{\tilde{r}, s}^{(3, J+1)} w_{2}^{t i m e}(t) w_{\tilde{r}}^{r e c}
$$

where the time window $w_{2}$ is now tighter than before, to just include the length of the signal, $5 / f_{0}$, assuming a 5-cycle toneburst. This can be adjusted to match the signal length as appropriate. Subsequently a filter is applied

$$
{\overline{\phi^{(5, J+1)}}}_{q, s}= \begin{cases}{\overline{\phi^{(4, J+1)}}}_{q, s} & |q| \leq G_{2} \\ 0 & |q|>G_{2}\end{cases}
$$

At the end of the process, the signals are quite clean so only the DRFT components which are clearly not part of it should be removed (rather than before, where just the components which were very likely to be part of it were needed); for the configuration in this paper $G_{2}=25$ was used. This windowed, filtered result is then forward-propagated and taken as the final separated signal.

It should be noted that, by reciprocity, it is possible to select a single receiver and perform the same separation algorithm across the different sources. However, it has been found that this does not significantly enhance the ability to select particular wavefronts so it has not been considered further.

\section{VALIDATION}

A photograph of the experimental configuration used to demonstrate the separation algorithm is shown in Fig. 5(a). A $2 \mathrm{~m}$ long section of schedule 40 12" pipe (outer diameter $323.85 \mathrm{~mm}$, wall thickness 
$10.312 \mathrm{~mm}$ ) had EMAT transducers placed in two rings with a separation distance of $800 \mathrm{~mm}$. These transducers are described in [30], are omnidirectional, operate at $50 \mathrm{kHz}$ and are designed to excite $\mathrm{A}_{0}$ waves; these have a wavelength of $38 \mathrm{~mm}$. $N=24$ transducers are used in each ring of the array, which results in a transducer separation distance of $42.4 \mathrm{~mm}$ or $1.12 \lambda$, significantly above the $\lambda / 2$ Nyquist requirement, making the system subsampled and potentially leading to aliasing. A defect is machined into the pipe; this is an axisymmetric raised-cosine shape, with width $180 \mathrm{~mm}$ and depth $3 \mathrm{~mm}$.

Figure 5(b) presents the time traces across the 24 receivers for source 8 providing the illumination. Firstly the crosstalk at zero time was removed by windowing the signals and dispersion correction was applied, as shown in Fig. 5(c). Figure 5(d) shows this replicated, and it is noticeable that there are some strong signals appearing at around $2 \mathrm{~m}$; these are caused by reflections from the ends of the pipe. Figure 5(e) shows the separated time traces, achieved by the process described in Sec. II-B, confirming that the approach is robust to experimental uncertainties. Figure 5(f) shows the separated traces for receiver 14 with source 8 ; the three distinct orders can be easily separated by time windowing. Figure $5(\mathrm{~g})$ presents a more complex example, for receiver 9 with source 8; here the second and third helical arrivals overlap to a large extent; despite this they are separated well by the algorithm. In this case they cause destructive interference in the combined signal, which is why the Hilbert envelope shown has an amplitude lower than that of either constituent signal. For this configuration, the helical path separation algorithm took around 17 seconds on an HP Z820 workstation with two 8-core Intel Xeon CPU E5-2665 processors running at $2.40 \mathrm{GHz}$; it should be noted, however, that this is for a relatively unoptimised Matlab version and if further speed improvement is needed alternative languages may well enable better performance. It should be noted that the highly parallel nature of the problem (here a total of 2880 traces should be separated) and the repeated use of discrete Fourier transforms suggest the architecture of a graphics card would be well suited to this problem.

Figure 6 presents images from the separated time traces. Figure 6(a) gives the defect shape, the Hannshaped axisymmetric defect described above. Reconstructions are performed from the experimental data illustrated in Fig. 5(b). Initially, just the first arrivals are used to generate the reconstruction shown in Fig. 6(b), which demonstrates what can be achieved without using helical path separation. Here, the VISCIT (Virtual Image Space Component Iterative Technique) approach [31] is used with HARBUT (Hybrid Algorithm for Robust Breast Ultrasound Tomography) [32] to compensate for the missing data, but even so, the defect depth is clearly underestimated by the reconstruction, and is very elongated. By 


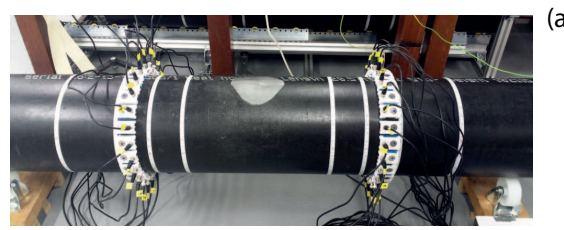

(a)

(b)
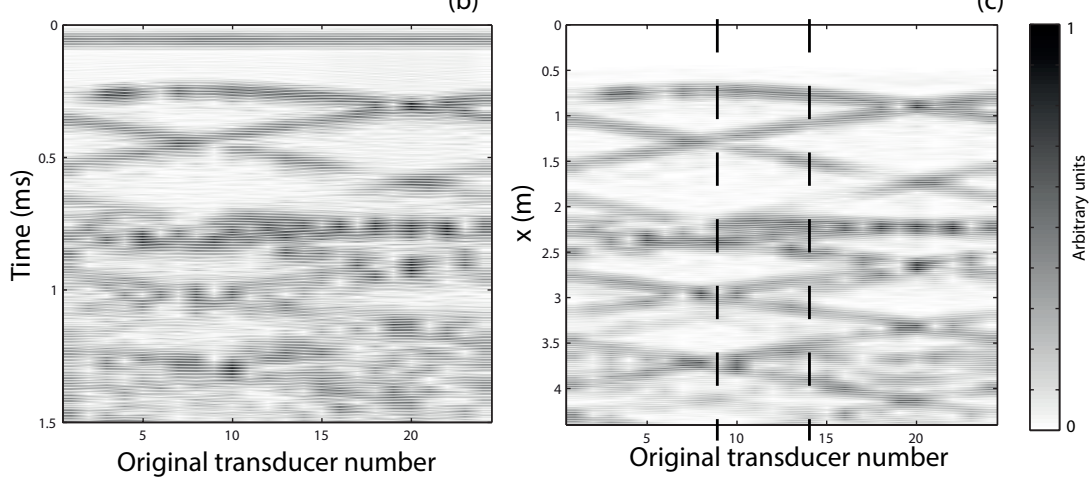

(d)
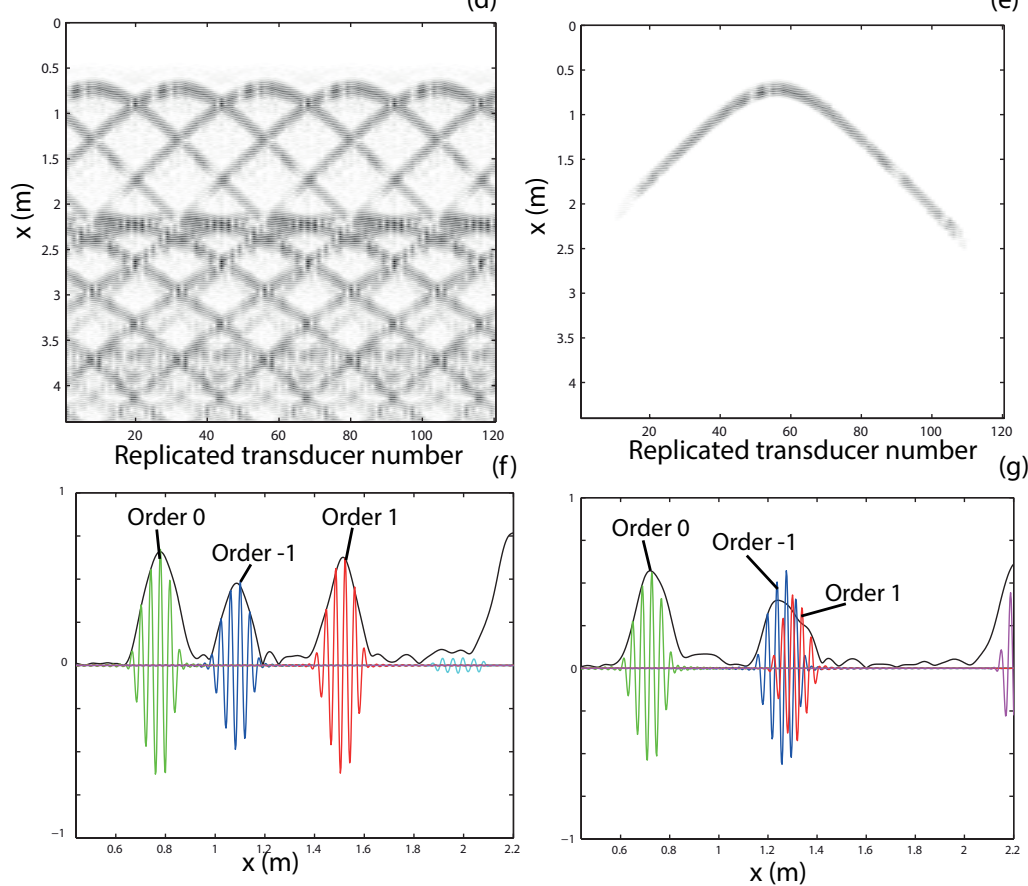

Figure 5. Media-Color 2 Separation of the experimental time traces. (a) shows a photo of the experimental setup. (b) shows the original signals received across the receiver array for source 8 of 24. In (c) the crosstalk has been removed and dispersion correction applied to the signals, enabling the wavepackets to be seen more clearly. (d) replicates the signals five times (for five orders) and (e) shows the separated signals extracted by the process in Sec. III. (f) plots the separated signal for receiver 14 with source 8; the Hilbert envelope of the original signal of (c) is included for reference. (g) is the same as (f) except for receiver 9 with source 8 , where the overlapping signals have been successfully separated. The different colours here are used to signify different modes. The signals for (f) and (g) are marked with dashed lines in (c). 

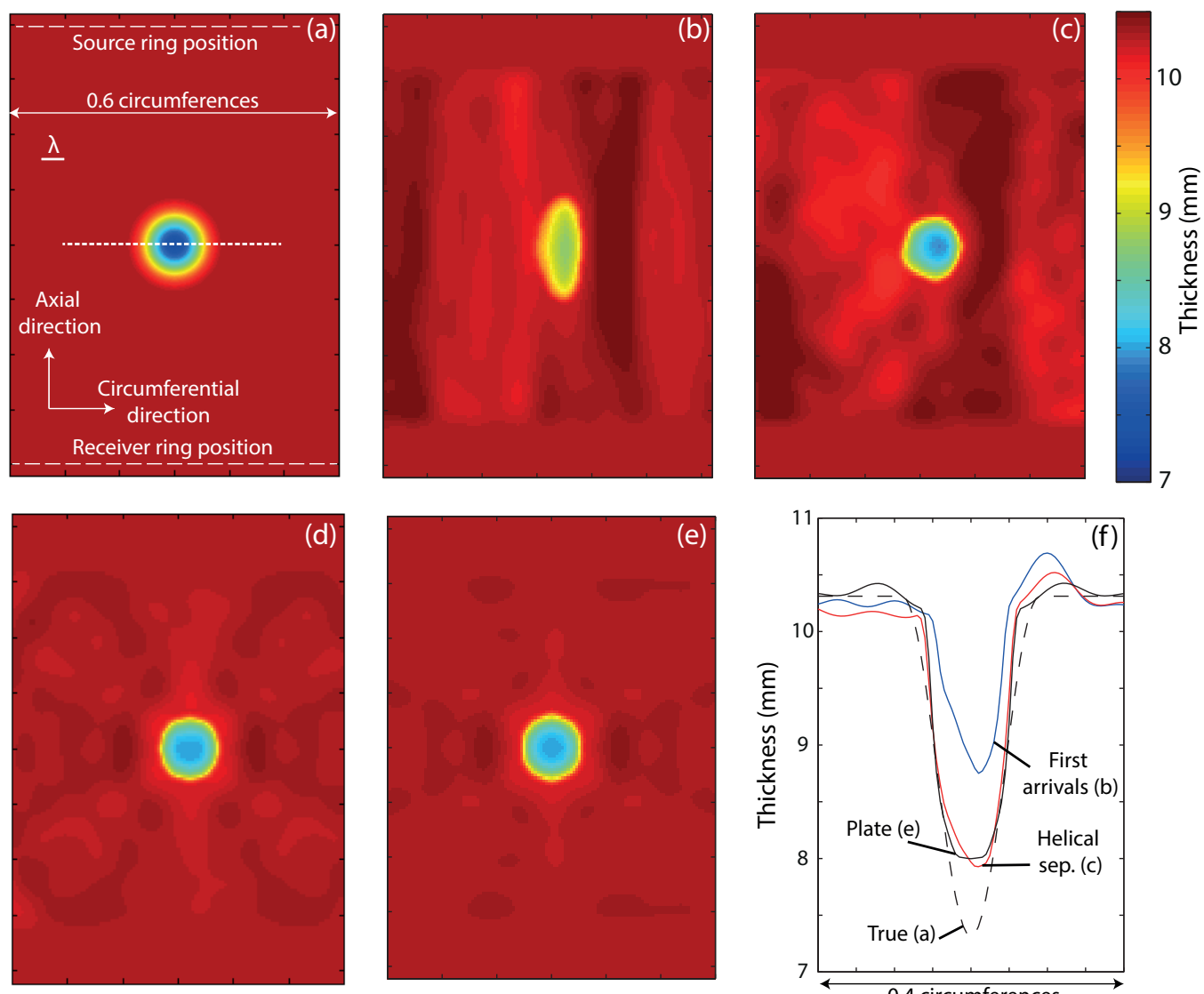

Figure 6. Media-Color 3 Images from the separated helical paths. (a) shows the test defect, a 3mm deep, 180mm wide Hannshaped axisymmetric defect. (b) presents the thickness reconstruction if no helical path separation was performed, i.e. just the first arrivals were used in the reconstruction, from the experimental data in Fig. 5(c). (c) presents the case when the helical path separated data of Fig. 5(e) was used to generate the image. (d) shows the results from an equivalent FE model of the pipe, again with the helical path separation algorithm used, and (e) shows the output from an equivalent FE model of a flat plate, thus avoiding the need for helical path separation and providing a 'theoretical maximum' baseline for comparison; if the helical path separation worked perfectly, the results for (d) and (e) would match. (f) plots cross sections along the line marked in (a).

contrast Fig. 6(c) shows the results (again generated by HARBUT with VISCIT) from the same data when the helical paths are separated using the approach outlined here, producing the data shown in Fig. 5(e). The shape of the defect shows no evidence of the elongation, and the maximum depth is much closer to the true depth. The remaining offset in maximum depth is a result of the remaining missing angles which cannot be recovered from the helical path separation approach and work is ongoing to develop the imaging algorithms to address this. To confirm the errors in the reconstruction are a result of the imaging algorithm rather than the helical separation approach, a comparison will be performed with 'ideal' finite 
element (FE) data.

An FE model of the pipe was generated, with linear 3D brick elements $1 \mathrm{~mm}$ in size, i.e. around 30-40 per wavelength, with 12 elements through the wall thickness. The pipe was meshed using a uniform, structured mesh, and the defect introduced using the approach discussed in [26], where the mesh was compressed in the normal direction to the surface to generate the desired change in thickness. This 46.5M degrees-of-freedom model was produced through a Matlab script, and solved in parallel on four graphics cards (6GB Nvidia GTX Titans with 336GB/sec bandwidth) using the Pogo solver [33]; this enabled the full set of 24 illuminations corresponding to 24 sources to be simulated in around 18 hours. Figure 6(d) provides the resulting image from this data set, having had the helical paths separated, and it encouragingly appears very similar to the experimental equivalent in Fig. 6(c). For comparison, a 'theoretical maximum' case is defined, which represents what the reconstruction would look like if the helical orders could be perfectly separated. To do this, the FE model of the pipe was unwrapped and flattened into a plate, and extended in the former circumferential direction to enable an equivalent array length to that which can be obtained from the helical path separation algorithm. An image from this is shown in Fig. 6(e), and a comparison of cross sections is shown in Fig. 6(f). From this, it is very encouraging that the images from the helical path separation algorithm, shown in Figs. 6(c) and 6(d), match the theoretical maximum closely, providing compelling evidence that the algorithm is successfully separating the helical paths without introducing any significant errors.

For further demonstration of performance, a different, deeper, defect has been considered, as shown in Fig. 7. This is generated using the same raised-cosine function from before, scaled to make it $120 \mathrm{~mm}$ wide and $6 \mathrm{~mm}$ deep. The helical path separation algorithm has again performed well, with very few visible artefacts in either the experimental results Fig. 7(a) or from an equivalent finite element model Fig. 7(b). Fig. 7(c) shows good agreement between both the experimental and numerical examples. Both curves do underestimate wall loss compared to the true map, but as demonstrated for the $3 \mathrm{~mm}$ deep case above, this is caused by the reconstruction algorithm, not the helical separation approach.

\section{CONCLUSION}

This paper has presented a new technique to enable guided waves travelling in helical paths on a pipe to be separated in an approach which is robust to experimental uncertainties and undersampling. The technique involves backpropagating received signals to the source such that they are approximately aligned, then extracting the desired orders by an iterative filtering approach. This was applied to exper- 


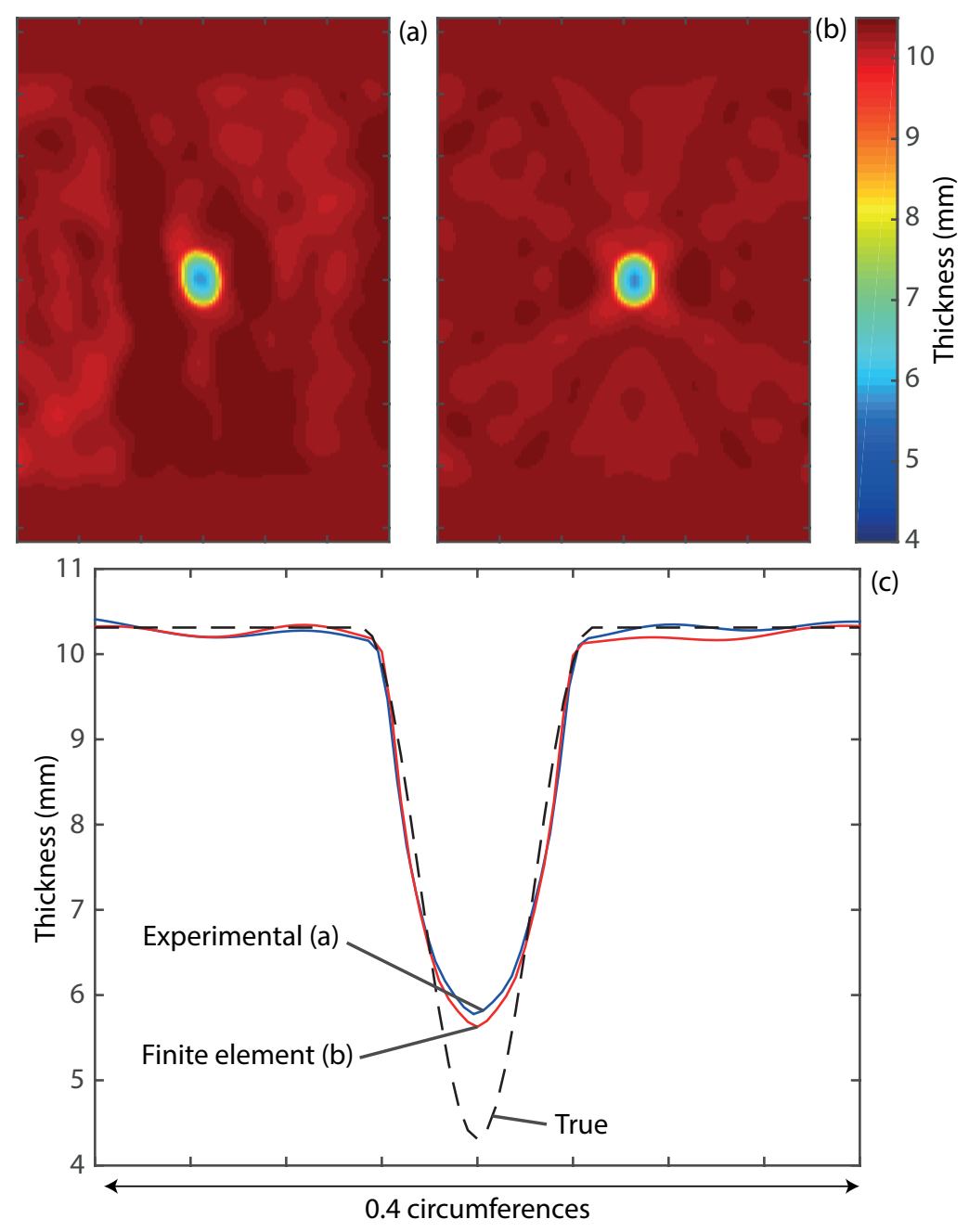

Figure 7. Media-Color 4 Results for a $6 \mathrm{~mm}$ deep defect, of diameter $120 \mathrm{~mm}$, generated using the same raise-cosine function as illustrated in Fig. 6(a), and with the same pipe and transducer configuration. (a) shows the results for the experimental data, separated by the algorithm outlined here. (b) shows the equivalent from a finite element model. (c) gives the cross sections through the two reconstructions, compared to the true thickness profile.

imental test data, separating the wavepackets well and enabling a clean image to be produced from the resulting data, and was achieved despite the presence of experimental uncertainties such as noise and transducer mispositioning.

The technique has been demonstrated on a simple cylindrical pipe, but the backpropagation approach would enable more general configurations such as pipes featuring bends to be considered, assuming the geometry is known. Similarly, different transducer configurations could be used, since the backpropagation stage will align the signals. Additionally, the method could be developed as an iterative technique, where 
an intermediate approximate image would allow improved backpropagation, allowing the separation to be improved at the next step. However, given the results are good without resorting to these approaches, it is unlikely that much benefit could be achieved from this.

\section{ACKNOWLEDGEMENTS}

This work was supported by EPSRC grants EP/H040072/1 and EP/H045430/1 with contributions from Petrobras and Shell. The authors are grateful to Prof. Peter Cawley and Prof. Mike Lowe for the critical reading and helpful comments.

\section{REFERENCES}

[1] R. Wagner, O. Goncalves, A. Demma, and M. Lowe, "Guided wave testing performance studies: comparison with ultrasonic and magnetic flux leakage pigs," Insight-Non-Destructive Testing and Condition Monitoring, vol. 55, no. 4, pp. 187-196, 2013.

[2] D. Jansen and D. Hutchins, "Lamb wave tomography," in Ultrasonics Symposium, 1990. Proceedings., IEEE 1990, pp. 1017-1020, IEEE, 1990.

[3] B. Hildebrand, T. Davis, G. Posakony, and J. Spanner, "Lamb wave tomography for imagimg erosion/corrosion in piping," in Review of Progress in Quantitative Nondestructive Evaluation, pp. 967-973, Springer, 1999.

[4] F. Jenot, M. Ouaftouh, M. Duquennoy, and M. Ourak, "Corrosion thickness gauging in plates using Lamb wave group velocity measurements," Measurement Science and Technology, vol. 12, pp. 1287-1293, Aug. 2001.

[5] K. R. Leonard and M. K. Hinders, "Guided wave helical ultrasonic tomography of pipes," The Journal of the Acoustical Society of America, vol. 114, no. 2, pp. 767-774, 2003.

[6] L. R. F. Rose and C. H. Wang, "Mindlin plate theory for damage detection: imaging of flexural inhomogeneities.," The Journal of the Acoustical Society of America, vol. 127, pp. 754-63, Feb. 2010.

[7] A. Volker and J. Bloom, "Experimental results of guided wave travel time tomography," in AIP Conference Proceedings, vol. 1335 , p. $215,2011$.

[8] P. Huthwaite and F. Simonetti, "High-resolution guided wave tomography," Wave Motion, vol. 50, no. 5, pp. 979 - 993, 2013.

[9] J. McKeon and M. Hinders, "Parallel projection and crosshole lamb wave contact scanning tomography," The Journal of the Acoustical Society of America, vol. 106, p. 2568, 1999.

[10] J. Pei, M. Yousuf, F. Degertekin, B. Honein, and B. Khuri-Yakub, "Lamb wave tomography and its application in pipe erosion/corrosion monitoring," Research in Nondestructive Evaluation, vol. 8, pp. 189-197, 1996. 10.1007/BF02433949.

[11] E. Malyarenko and M. Hinders, "Fan beam and double crosshole lamb wave tomography for mapping flaws in aging aircraft structures," The Journal of the Acoustical Society of America, vol. 108, p. 1631, 2000.

[12] K. Leonard and M. Hinders, "Multi-mode lamb wave tomography with arrival time sorting," The Journal of the Acoustical Society of America, vol. 117, p. 2028, 2005.

[13] A. Volker and J. Bloom, "Guided wave travel-time tomography for bends," in AIP Conference Proceedings, vol. 1335, p. 223, 2011. 
[14] C. Willey, F. Simonetti, P. Nagy, and G. Instanes, "Guided wave tomography of pipes with high-order helical modes," NDT \& E International, vol. 65, pp. 8-21, 2014.

[15] A. H. Rohde, M. Veidt, L. R. F. Rose, and J. Homer, "A computer simulation study of imaging flexural inhomogeneities using plate-wave diffraction tomography.," Ultrasonics, vol. 48, pp. 6-15, Mar. 2008.

[16] P. Belanger, P. Cawley, and F. Simonetti, "Guided wave diffraction tomography within the born approximation," Ultrasonics, Ferroelectrics and Frequency Control, IEEE Transactions on, vol. 57, no. 6, pp. 1405-1418, 2010.

[17] J. Rose and J. Barshinger, "Using ultrasonic guided wave mode cutoff for corrosion detection and classification," in Ultrasonics Symposium, 1998. Proceedings., 1998 IEEE, vol. 1, pp. 851-854 vol.1.

[18] P. Belanger, "High order shear horizontal modes for minimum remnant thickness.," Ultrasonics, vol. 54, pp. 1078-87, Apr. 2014.

[19] K. R. Leonard and M. K. Hinders, “Lamb wave tomography of pipe-like structures," Ultrasonics, vol. 43, no. 7, pp. 574$583,2005$.

[20] P. B. Nagy, F. Simonetti, and G. Instanes, "Corrosion and erosion monitoring in plates and pipes using constant group velocity lamb wave inspection," Ultrasonics, 2014.

[21] A. Volker and T. van Zon, "1-d profiling using highly dispersive guided waves," AIP Conference Proceedings, vol. 1581, no. 1, pp. 186-192, 2014.

[22] K. S. Ho, D. R. Billson, and D. A. Hutchins, "Ultrasonic Lamb wave tomography using scanned EMATs and wavelet processing," Nondestructive Testing and Evaluation, vol. 22, pp. 19-34, Mar. 2007.

[23] J. Hou, K. Leonard, and M. Hinders, "Automatic multi-mode lamb wave arrival time extraction for improved tomographic reconstruction," Inverse Problems, vol. 20, no. 6, p. 1873, 2004.

[24] A. Raghavan and C. E. S. Cesnik, "Guided-wave signal processing using chirplet matching pursuits and mode correlation for structural health monitoring," Smart Materials and Structures, vol. 16, pp. 355-366, Apr. 2007.

[25] J. E. Michaels, S. J. Lee, J. S. Hall, and T. E. Michaels, "Multi-mode and multi-frequency guided wave imaging via chirp excitations," in SPIE Smart Structures and Materials + Nondestructive Evaluation and Health Monitoring (T. Kundu, ed.), pp. 79840I-79840I-11, International Society for Optics and Photonics, Mar. 2011.

[26] P. Huthwaite, "Evaluation of inversion approaches for guided wave thickness mapping," Proceedings of the Royal Society A: Mathematical, Physical and Engineering Science, vol. 470, no. 2166, p. 20140063, 2014.

[27] G. Liu and J. Qu, "Guided Circumferential Waves in a Circular Annulus," Journal of Applied Mechanics, vol. 65, p. 424, June 1998.

[28] A. J. Brath, F. Simonetti, P. B. Nagy, and G. Instanes, "Acoustic formulation of elastic guided wave propagation and scattering in curved tubular structures," IEEE Transactions on Ultrasonics, Ferroelectrics, and Frequency Control, vol. 61, pp. 815-829, May 2014.

[29] P. D. Wilcox, "A rapid signal processing technique to remove the effect of dispersion from guided wave signals," Ultrasonics, Ferroelectrics and Frequency Control, IEEE Transactions on, vol. 50, no. 4, pp. 419-427, 2003.

[30] M. Seher, P. Huthwaite, M. Lowe, P. Nagy, and P. Cawley, "Numerical design optimization of an EMAT for A0 Lamb wave generation in steel plates," AIP Conference Proceedings, vol. 1581, no. 1, pp. 340-347, 2014.

[31] P. Huthwaite, A. A. Zwiebel, and F. Simonetti, "A new regularization technique for limited-view sound-speed imaging.," IEEE transactions on ultrasonics, ferroelectrics, and frequency control, vol. 60, no. 3, pp. 603-613, 2013.

[32] P. Huthwaite and F. Simonetti, "High-resolution imaging without iteration: a fast and robust method for breast ultrasound tomography," Journal of the Acoustical Society of America, vol. 130, no. 3, pp. 1721-1734, 2011. 
[33] P. Huthwaite, "Accelerated finite element elastodynamic simulations using the GPU," Journal of Computational Physics, vol. 257, Part A, pp. 687 - 707, 2014. 